\title{
CARACTERIZAÇÃO DAS PROPRIEDADES FÍSICO-QUÍMICAS DA POLPA DA MACAÚBA (ACROCOMIA ACULEATA) APÓS DIFERENTES TRATAMENTOS PÓS-COLHEITA E ARMAZENAMENTO.
}

\author{
L. A. L. QUEIROZ ${ }^{1}$, C. S. NASCIMENTO1, A. L. M. SILVEIRA ${ }^{1}$, R. M. FONSECA ${ }^{1}$, E. C. \\ $\mathrm{CREN}^{1}$ e M. H. C. ANDRADE ${ }^{1}$ \\ 1 Universidade Federal de Minas Gerais, Escola de Engenharia, Departamento de Engenharia \\ Química \\ E-mail: lorenaqueiroz84@yahoo.com.br
}

\begin{abstract}
RESUMO - Os frutos da macaúba podem ser promissora fonte de óleo vegetal para fins alimentícios, cosméticos e energia, devido à elevada produtividade e a obtenção de dois tipos de óleo. Contudo, a principal restrição está associada com a rápida deterioração do fruto e seus produtos óleo e torta. Assim a preservação da qualidade dos frutos necessita do desenvolvimento de técnicas adequadas, que se iniciam na coleta, passam pelo tratamento pós-colheita, armazenamento, preparo e extração dos óleos. O objetivo deste trabalho foi identificar condições de tratamento pós-colheita capazes de promover a preservação do fruto durante o período de 30 dias, por meio da avaliação da umidade, teor de óleo e acidez titulável como indicativo da qualidade da polpa do fruto. Seis tipos de tratamento foram testados e os resultados obtidos em três safras indicam que o tratamento mais recomendável é a lavagem com água seguida por rápida imersão em solução clorada $2 \%$ e posterior secagem em estufa a $60^{\circ} \mathrm{C}$ por $24 \mathrm{~h}$.
\end{abstract}

\section{INTRODUÇÃO}

Os óleos vegetais, além de consumidos diretamente na alimentação, constituem-se em importante matéria- prima para a formulação de biodiesel, alimentos, cosméticos, fármacos e lubrificantes.

O Brasil possui uma enorme diversidade de espécies vegetais oleaginosas das quais se podem extrair óleos, e destaca-se a macaúba (Acrocomia aculeata), uma palmeira típica do Cerrado brasileiro que produz um fruto capaz de ser utilizado como fonte de óleo vegetal com valor agregado (SILVA e CAÑO-ANDRADE, 2011).

O produto economicamente mais representativo da palmeira é o fruto. Tanto da polpa como da amêndoa podem ser obtidos óleos, e as tortas geradas pela sua extração podem ser usadas como ração animal, devido seu elevado valor proteico, ou ainda como ingredientes para formulação de alimentos como bolos, biscoitos, cookies e outros. 
Segundo Cetec (1983), a macaúba tem capacidade de produção entre 1500 e $5000 \mathrm{~kg}$ de óleo/hectare. Seu aproveitamento em grande escala tem se dirigido principalmente à utilização do óleo da polpa visando à produção de biodiesel.

A colheita da macaúba é concentrada nos meses de novembro a janeiro (pico da safra) podendo se estender por mais dois meses dependendo da região e do clima, o que torna necessário um sistema eficiente de estocagem, para permitir que a usina de beneficiamento opere em um regime mais adequado. Sendo que, o sistema de estocagem irá definir a qualidade do óleo produzido principalmente em relação à acidez (ARAÚJO, 2011).

O óleo da macaúba apresenta ótima qualidade, se comparado a outras oleaginosas, entretanto a etapa de pós-colheita do fruto é ainda muito pouco estudada e a extração do óleo é feita aplicando-se tecnologias adaptadas de outras oleaginosas. Estes fatores contribuem para a baixa qualidade do óleo. Estudos sobre técnicas de colheitas e tratamentos pós-colheita visando armazenar os frutos com qualidade são necessários para se obter avanços no processamento do fruto da macaúba, permitindo assim o uso do óleo para seus diversos fins, inclusive finalidades nobres (MOTA et al, 2011).

Diante deste contexto, o objetivo deste trabalho foi identificar condições de tratamento póscolheita capazes de promover a preservação do fruto durante o período de 30 dias, por meio da avaliação da umidade, teor de óleo e acidez titulável como indicativo da qualidade da polpa do fruto.

\section{MATERIAL E MÉTODOS}

\subsection{Safra 2011/2012}

Os frutos da macaúba foram obtidos de palmeiras selecionadas no campus da Universidade Federal de Minas Gerais - Belo Horizonte. Os frutos ao atingirem ponto de maturação ideal se desprendem do cacho e caem ao chão, podendo ocorrer rachaduras e contaminação por microrganismos, levando principalmente à deterioração e a alteração na qualidade da polpa e consequentemente dos produtos que podem ser obtidos da mesma. Assim, com o intuito de impedir esse tipo de contaminação dos frutos, ao redor de cada palmeira selecionada, foram instalados coletores construídos em estrutura de PVC e redes de pescaria, distanciados do solo por cerca de meio metro.

Para verificar o efeito de diferentes tratamentos pós-colheita os frutos foram separados em seis diferentes lotes: lote 0-frutos caídos ao chão, armazenados sem limpeza prévia; lote 1-frutos caídos no coletor, armazenados sem limpeza prévia; lote 2-frutos caídos no coletor, lavados com água, secos à temperatura ambiente; lote 3-frutos caídos no coletor, lavados e higienizados por imersão em solução de hipoclorito de sódio $2 \%$, secos à temperatura ambiente; lote 4-frutos caídos no coletor, secos na estufa a $60^{\circ} \mathrm{C}$ por $24 \mathrm{~h}$; lote 5- frutos caídos no coletor, lavados e higienizados por imersão em solução de hipoclorito de sódio $2 \%$ e secos na estufa a $60^{\circ} \mathrm{C}$ por 24 h. 


\subsection{Safra 2012/2013}

Nesta safra foi coletado um cacho inteiro em uma palmeira na área rural de Taquaraçu de Minas (MG), em um período que os frutos estavam de desprendendo naturalmente do cacho. Apenas os tratamentos que obtiveram os melhores resultados na safra anterior foram repetidos, porém com algumas adaptações: lote 1-frutos coletados no cacho, armazenados sem limpeza prévia; lote 2-frutos coletados no cacho, lavados com água, imersos rapidamente em solução de hipoclorito de sódio $2 \%$ e secos à temperatura ambiente; lote 3-frutos coletados no cacho, lavados com água, imersos rapidamente em solução de hipoclorito de sódio $2 \%$ e secos na estufa a $60^{\circ} \mathrm{C}$ por $24 \mathrm{~h}$.

\subsection{Safra 2013/2014}

Os frutos foram coletados novamente no campus da UFMG-Belo Horizonte, porém devido ao resultado obtido na primeira safra não foi utilizado o coletor. Sendo assim, os frutos foram divididos da seguinte forma: lote 0-frutos coletados no chão, armazenados sem limpeza prévia; lote 2 -frutos coletados no chão, lavados com água, imersos rapidamente em solução de hipoclorito de sódio $2 \%$ e secos à temperatura ambiente; lote 3-frutos coletados no chão, lavados com água, imersos rapidamente em solução de hipoclorito de sódio $2 \%$ e secos na estufa a $60^{\circ} \mathrm{C}$ por $24 \mathrm{~h}$.

Todos os lotes foram armazenados à temperatura ambiente, dentro de cestos plásticos com furos. As análises foram feitas no dia da coleta, após o tratamento escolhido para cada lote (tempo 0) e após 30 dias (tempo 30). Sendo assim, foram utilizados na faixa de 15 a 20 frutos (50\%) em cada tempo avaliado.

\subsection{Análises físico-químicas}

A determinação do teor de umidade e voláteis (doravante umidade) foi realizada segundo as normas da American Oil Chemists' Society (AOCS), método Ac 2-41, que estabelece a secagem em estufa na temperatura de $130^{\circ} \mathrm{C}$ por três horas. $\mathrm{O}$ teor de óleo, em base seca, foi quantificado através da extração contínua de lipídios no aparelho de Soxhlet, utilizando éter de petróleo como solvente, de acordo com a normas da AOCS, método Bc 3-49. A acidez titulável, expressa em percentual da relação volume por massa, foi determinada através de titulações com solução de hidróxido de sódio 0,1 M, de acordo com as normas do Instituto Adolf Lutz, método 310/IV.

Todas as análises foram realizadas em triplicata. Cada tratamento teve seu valor médio calculado. Para comparação das médias foi aplicado o teste de Tukey a 10\% de probabilidade.

\section{RESULTADOS E DISCUSSÃO}

A variação do teor de umidade e da acidez titulável da polpa dos frutos coletados na safra 2011/2012, após os 30 dias de armazenamento, estão apresentados na Tabela 1.

No tempo "0", deve-se ressaltar que os valores de teor de umidade dos lotes 3 e 5 , onde houve a higienização por imersão em solução de hipoclorito de sódio, foram numericamente sempre maiores do que as umidades dos lotes que sofreram tratamentos semelhantes porém sem imersão, lotes 2 e 4 , o que pode indicar que o processo de higienização por imersão propicia uma 
absorção de umidade pelo fruto. Ainda, os resultados dos lotes 4 e 5, que passaram pela secagem, apresentam uma redução do teor de umidade da ordem de $20 \%$.

Tabela 1 - Variação do teor de umidade e acidez titulável da polpa da Macaúba

(safra 2011/2012)

\begin{tabular}{c|c|cc|cc}
\hline \multicolumn{2}{c|}{} & \multicolumn{2}{|c|}{$\begin{array}{c}\text { Teor de umidade } \\
(\%)\end{array}$} & \multicolumn{2}{c}{$\begin{array}{c}\text { Acidez titulável } \\
(\% \mathrm{v} / \mathrm{m})\end{array}$} \\
\hline \hline \multirow{3}{*}{$1^{\mathrm{a}}$} & Lote & Tempo 0 & Tempo 30 & Tempo 0 & Tempo 30 \\
\cline { 2 - 6 } coleta & 0 & $40,58^{\mathrm{abA}} \pm 1,05$ & $11,07^{\mathrm{B}} \pm 4,59$ & $4,77^{\mathrm{aA}} \pm 0,33$ & $21,81^{\mathrm{B}} \pm 1,26$ \\
\hline \multirow{2}{*}{$2^{\mathrm{a}}$} & 1 & $38,70^{\mathrm{bA}} \pm 1,07$ & $9,48^{\mathrm{B}} \pm 1,16$ & $3,95^{\mathrm{abA}} \pm 0,24$ & $18,48^{\mathrm{B}} \pm 0,62$ \\
coleta & 0 & $42,23^{\mathrm{abA}} \pm 2,22$ & $7,49^{\mathrm{B}} \pm 0,66$ & $1,29^{\mathrm{cA}} \pm 0,12$ & $19,0^{\mathrm{B}} \pm 0,19$ \\
& 2 & $39,31^{\mathrm{ab}} \pm 1,99$ & $8,97^{\mathrm{B}} \pm 0,6$ & $1,77^{\mathrm{cA}} \pm 0,11$ & $15,13^{\mathrm{B}} \pm 0,41$ \\
& 3 & $42,74^{\mathrm{aA}} \pm 2,02$ & $7,95^{\mathrm{B}} \pm 0,75$ & $1,50^{\mathrm{cA}} \pm 0,11$ & $23,93^{\mathrm{B}} \pm 0,47$ \\
\hline \multirow{2}{*}{$3^{\mathrm{a}}$} & 0 & $41,75^{\mathrm{aA}} \pm 1,01$ & $7,86^{\mathrm{B}} \pm 0,47$ & $1,46^{\mathrm{cA}} \pm 0,11$ & $35,27^{\mathrm{B}} \pm 0,08$ \\
coleta & 4 & $31,86^{\mathrm{cA}} \pm 0,75$ & $6,61^{\mathrm{B}} \pm 0,18$ & $1,79^{\mathrm{cA}} \pm 0,33$ & $8,51^{\mathrm{B}} \pm 0,22$ \\
& 5 & $32,85^{\mathrm{cA}} \pm 1,66$ & $5,56^{\mathrm{B}} \pm 0,16$ & $3,66^{\mathrm{bA}} \pm 0,75$ & $6,47^{\mathrm{B}} \pm 0,66$ \\
\hline
\end{tabular}

$\mathrm{a}, \mathrm{b}, \ldots . .$. (coluna) médias seguidas pela mesma letra não diferem pelo teste de Tukey $(\mathrm{p}<0,10)$.

$\mathrm{A}, \mathrm{B}, \ldots .$. (linha) médias seguidas por letras diferentes diferem pelo teste de Tukey $(\mathrm{p}<0,10)$.

Os frutos da primeira coleta apresentaram um maior teor de acidez titulável, o que indica que os frutos podem apresentar características diferentes a cada coleta. Ainda nessa coleta, nota-se que a utilização do coletor não influenciou no valor do teor de acidez titulável da polpa, uma vez que de acordo com o teste de Tukey, os valores não apresentaram diferença significativa.

Independente do tratamento pós-colheita recebido, verifica-se que após os 30 dias de armazenamento houve uma redução do teor de umidade, entre 72 a $83 \%$, e aumento da acidez titulável de até $2315 \%$.

A variação do teor de óleo da macaúba na safra 2011/2012 está apresentada na Tabela 2.

Tabela 2 - Variação do teor de óleo (\% em base seca) - safra 2011/2012

\begin{tabular}{cccc}
\hline & Lote & Tempo 0 & Tempo 30 \\
\hline \hline \multirow{2}{*}{$1^{\mathrm{a}}$ coleta } & 0 & $53,39^{\mathrm{abcA}} \pm 0,74$ & $62,93^{\mathrm{B}} \pm 0,87$ \\
& 1 & $49,79^{\mathrm{dA}} \pm 1,03$ & $58,86^{\mathrm{B}} \pm 3,03$ \\
\hline \multirow{3}{*}{$2^{\mathrm{a}}$ coleta } & 0 & $56,88^{\mathrm{aA}} \pm 0,73$ & $65,77^{\mathrm{B}} \pm 0,34$ \\
& 2 & $55,69^{\mathrm{abA}} \pm 0,92$ & $63,87^{\mathrm{B}} \pm 1,32$ \\
& 3 & $53,89^{\mathrm{abcA}} \pm 0,79$ & $56,15^{\mathrm{A}} \pm 1,71$ \\
\hline \multirow{3}{*}{$3^{\mathrm{a}}$ coleta } & 0 & $53,98^{\mathrm{abcA}} \pm 2,15$ & $54,17^{\mathrm{A}} \pm 7,4$ \\
& 4 & $51,58^{\mathrm{cdA}} \pm 2,69$ & $61,82^{\mathrm{B}} \pm 1,08$ \\
& 5 & $52,97^{\mathrm{bcdA}} \pm 0,29$ & $60,75^{\mathrm{B}} \pm 0,9$ \\
\hline
\end{tabular}

a,b, .....(coluna) médias seguidas pela mesma letra não diferem pelo teste de Tukey $(\mathrm{p}<0,10)$.

$\mathrm{A}, \mathrm{B}, \ldots . .($ linha) médias seguidas por letras diferentes diferem pelo teste de Tukey $(\mathrm{p}<0,10)$. 
Não se pode afirmar que os diferentes tratamentos pós-colheita utilizados proporcionaram o aumento ou a diminuição do teor de óleo na polpa, uma vez que não houve um padrão pelo teste de Tukey. Sendo assim, tornam-se necessários novos testes para verificar a influência dos tratamentos no teor de óleo da polpa. O teor de óleo da polpa dos lotes que não sofreram nenhum tratamento pós-colheita ( 0 e 1) variou de 49,79 a 53,98\%. Estes valores foram superiores aos encontrados por Ciconini et al. (2013) em frutos de quatro regiões distintas do estado de Mato Grosso do Sul, cujo teor médio de óleo em base seco foi de 25,07\%. Após 30 dias de armazenamento, o valor numérico do teor de óleo na polpa do fruto foi ligeiramente superior ao valor encontrado no tempo zero, contudo, os resultados do teste de Tukey para a comparação das médias indicaram que em alguns casos essa diferença não foi significativa.

Os resultados obtidos na safra 2012/2013 podem ser vistos nas Tabelas 3 e 4 .

Tabela 3 - Variação do teor de umidade e acidez titulável da polpa da Macaúba

(safra 2012/2013)

\begin{tabular}{c|cc|cc}
\hline & \multicolumn{2}{|c|}{$\begin{array}{c}\text { Teor de umidade } \\
(\%)\end{array}$} & \multicolumn{2}{c}{$\begin{array}{c}\text { Acidez titulável } \\
(\% \mathrm{v} / \mathrm{m})\end{array}$} \\
\hline \hline Lote & Tempo 0 & Tempo 30 & Tempo 0 & Tempo 30 \\
\hline 1 & $48,36^{\mathrm{bA}} \pm 0,85$ & $6,08^{\mathrm{B}} \pm 0,39$ & $0,43^{\mathrm{aA}} \pm 0,06$ & $15,78^{\mathrm{B}} \pm 0,25$ \\
2 & $51,01^{\mathrm{aA}} \pm 0,54$ & $5,76^{\mathrm{B}} \pm 0,38$ & $0,35^{\mathrm{aA}} \pm 0,05$ & $7,64^{\mathrm{B}} \pm 0,34$ \\
3 & $38,36^{\mathrm{cA}} \pm 0,17$ & $6,29^{\mathrm{B}} \pm 0,11$ & $1,09^{\mathrm{bA}} \pm 0,11$ & $5,41^{\mathrm{B}} \pm 0,48$ \\
\hline
\end{tabular}

$a, b, \ldots . .$. (coluna) médias seguidas pela mesma letra não diferem pelo teste de Tukey $(\mathrm{p}<0,10)$.

A, B,....(linha) médias seguidas por letras diferentes diferem pelo teste de Tukey $(\mathrm{p}<0,10)$.

No tempo "0" o teor de umidade da polpa apresentou diferença significativa em todos os tratamentos. Como esperado, houve redução da umidade após 30 dias de armazenamento na faixa de $83-89 \%$. Pode-se constatar que os teores de umidade dessa safra foram maiores que o da primeira, sendo que essa diferença pode ter ocorrido devido a dois fatores: os frutos foram coletados diretamente do cacho, havendo, portanto, a possibilidade de não estarem na maturação ideal; e diferentemente da primeira safra, o cacho foi coletado no município de Taquaraçu de Minas. Segundo Duarte et al. (2012) a umidade nos frutos de macaúba pode variar de acordo com o período de coleta, sendo que os frutos imaturos apresentam um maior teor de umidade. Há valores diversificados na literatura quanto ao teor de água na polpa da macaúba. Ciconini et al. (2013) obteve valores variando de 49,06\% a 63,00\% em frutos coletados tanto no bioma Cerrado como no bioma Pantanal, enquanto que os frutos do sertão Pernambucano, analisados por Oliveira et al. (2009) apresentaram umidade da polpa em torno de $41,85 \%$.

Verifica-se que o valor inicial da acidez titulável (tempo 0) do lote que passou por lavagem seguida pela secagem foi maior do que os demais tipos de tratamento, o que pode ser um indicativo de que esse processo propicia um aumento da acidez inicial da polpa. Considerando todos os lotes, após os trinta dias de armazenamento houve um aumento da acidez titulável entre 400 a $3500 \%$.

O percentual de óleo na polpa da safra 2012/2013, indicado na Tabela 4, variou entre $43,85 \%$ a $45,70 \%$ sendo menores que os valores obtidos na safra anterior. A maior umidade na 
polpa e o menor teor de óleo corroboram com a hipótese de que os frutos estavam imaturos quando o cacho foi retirado. Outra hipótese é de que a palmeira de Taquaraçu de Minas pertencia a uma subespécie diferente das palmeiras do campus da UFMG-Belo Horizonte. De acordo com Lira et al. (2013) a variabilidade genética, a região onde se encontra e condições edafoclimáticas são variáveis que determinam a quantidade de cada composição centesimal.

Tabela 4 - Variação do teor de óleo,\% em base seca (safra 2012/2013)

\begin{tabular}{ccc}
\hline Lote & Tempo 0 & Tempo 30 \\
\hline \hline 1 & $45,70^{\mathrm{aA}} \pm 2,30$ & $56,08^{\mathrm{B}} \pm 2,33$ \\
2 & $44,06^{\mathrm{aA}} \pm 0,54$ & $58,33^{\mathrm{B}} \pm 0,47$ \\
3 & $43,85^{\mathrm{aA}} \pm 0,61$ & $43,73^{\mathrm{A}} \pm 0,75$ \\
\hline
\end{tabular}

$\overline{a, b}, \ldots . .($ coluna) médias seguidas pela mesma letra não diferem pelo teste de Tukey $(\mathrm{p}<0,10)$.

$\mathrm{A}, \mathrm{B}, \ldots .$. (linha) médias seguidas por letras diferentes diferem pelo teste de Tukey $(\mathrm{p}<0,10)$.

A variação do teor de umidade e acidez titulável da polpa dos frutos coletados na safra 2013/2014 encontram-se na Tabela 5.

Tabela 5 - Variação do teor de umidade e acidez titulável da polpa da Macaúba

(safra 2013/2014)

\begin{tabular}{c|cc|cc}
\hline & \multicolumn{2}{|c|}{$\begin{array}{c}\text { Teor de umidade } \\
(\%)\end{array}$} & \multicolumn{2}{c}{$\begin{array}{c}\text { Acidez titulável } \\
(\% \mathrm{v} / \mathrm{m})\end{array}$} \\
\hline \hline Lote & Tempo 0 & Tempo 30 & Tempo 0 & Tempo 30 \\
\hline 0 & $44,78^{\mathrm{aA}} \pm 0,64$ & $22,60^{\mathrm{B}} \pm 0,73$ & $0,86^{\mathrm{bB}} \pm 0,07$ & $24,90^{\mathrm{A}} \pm 0,19$ \\
2 & $45,92^{\mathrm{aA}} \pm 0,73$ & $15,64^{\mathrm{B}} \pm 0,30$ & $0,87^{\mathrm{bB}} \pm 0,11$ & $21,30^{\mathrm{A}} \pm 0,70$ \\
3 & $38,49^{\mathrm{bA}} \pm 0,20$ & $13,14^{\mathrm{B}} \pm 0,39$ & $2,48^{\mathrm{aB}} \pm 0,07$ & $15,64^{\mathrm{A}} \pm 0,34$ \\
\hline
\end{tabular}

$\mathrm{a}, \mathrm{b}, \ldots . . .($ coluna) médias seguidas pela mesma letra não diferem pelo teste de Tukey $(\mathrm{p}<0,10)$.

$\mathrm{A}, \mathrm{B}, \ldots .$. (linha) médias seguidas por letras diferentes diferem pelo teste de Tukey $(\mathrm{p}<0,10)$.

O teor de umidade da polpa em relação aos diferentes tratamentos se comportou de forma semelhante ao das outras safras. $\mathrm{O}$ teor de umidade do lote 3 apresentou diferença estatística, sendo menor em torno de $15 \%$ comparado aos outros lotes, o que era esperado pois a secagem que o lote foi submetido tem justamento o intuito de reduzir a umidade. No lote 2, apesar do valor ser maior numericamente, não se observou diferença significativa pelo teste de Tukey, sendo assim, não se pode afirmar que a imersão na solução de hipoclorito de sódio acarreta em uma aumento da umidade da polpa.

A acidez da polpa em relação aos tratamentos apresentou comportamento semelhante das outras safras. Após os 30 dias de armazenamento a acidez titulável da polpa aumentou consideravelmente, entre $530 \%$ e $2800 \%$. Observa-se que o lote 3 foi o que apresentou o menor aumento da acidez em todas as coletas. Nas três safras consecutivas, os lotes que não passaram por nenhum tratamento pós-colheita tiveram os maiores aumentos de acidez, mesmo apresentando uma acidez inicial mais baixa. 
A variação do teor de óleo da polpa (safra 2013/2014) após o período de armazenamento pode ser vista na Tabela 6 .

Tabela 6 - Variação do teor de óleo, \% em base seca (safra 2013/2014)

\begin{tabular}{ccc}
\hline Lote & Tempo 0 & Tempo 30 \\
\hline \hline 1 & $49,58^{\mathrm{aB}} \pm 0,64$ & $64,94^{\mathrm{A}} \pm 1,08$ \\
2 & $48,99^{\mathrm{aB}} \pm 0,34$ & $63,91^{\mathrm{A}} \pm 1,64$ \\
3 & $48,54^{\mathrm{aB}} \pm 1,20$ & $50,94^{\mathrm{A}} \pm 0,51$ \\
\hline a,b, ....(coluna) médias seguidas pela mesma letra não diferem pelo teste de Tukey $(\mathrm{p}<0,10)$. \\
$\mathrm{A}, \mathrm{B}, \ldots$. (linha) médias seguidas por letras diferentes diferem pelo teste de Tukey $(\mathrm{p}<0,10)$.
\end{tabular}

Nota-se que na maior parte dos casos, após trinta dias de armazenamento houve um aumento significativo no teor de óleo da polpa. Este aumento do teor de óleo é relatado na literatura por diferentes autores. Martins (2013) também observou um incremento no teor de óleo do mesocarpo dos frutos da macaúba, tratados com radiação gama, durante o período de armazenamento, cujo ponto de conversão máxima foi em torno dos 30 dias, com maior acúmulo de óleo nos frutos que não foram tratados. Ainda segundo o autor, em frutos submetidos a diferentes combinações de temperatura e tempo de secagem ( 4 ou 8 dias), a influência exercida pelo tempo de armazenamento no acúmulo de óleo foi mais pronunciada nos frutos sem tratamento e nos secos a $45^{\circ} \mathrm{C}$ por 4 dias, sendo que o uso de tempo e temperatura maiores restringiram o acúmulo natural de óleo do mesocarpo durante o armazenamento. Em estudos realizados para avaliar a qualidade do óleo de macaúba em função do tempo de permanência no campo dentro de coletores, Souza (2013) observou que o teor de lipídeos da polpa aumentou com o tempo de permanência dos frutos no campo, tendo um ponto de máximo aos 11 dias, atingindo 40,35\%, levando-se a conclusão de que ocorre síntese de lipídeos após os frutos serem destacados do cacho.

\section{CONCLUSÃO}

Os resultados obtidos nas análises de três safras consecutivas indicam que se o objetivo for preservar os frutos da macaúba, de forma com que a polpa se mantenha com menor acidez, o tratamento mais recomendável é a lavagem com água seguida por rápida imersão em solução clorada $2 \%$ e posterior secagem em estufa a $60^{\circ} \mathrm{C}$ por $24 \mathrm{~h}$. Outro efeito a ser destacado, é o aumento no percentual de lipídeos durante o período de armazenamento.

\section{REFERENCIAS}

AOCS - AMERICAN OIL CHEMISTS SOCIETY. Official methods and recommended practices of the American Oil Chemists Society. Champaign, 2009.

ARAÚJO, M. M. Conhecendo a cadeia produtiva da macaúba: Projeto piloto do Alto ParnaíbaMG. Informe Agropecuário, Belo Horizonte: EPAMIG, v.32, n. 265, p. 31, nov./dez. 2011. 


\section{9 a 22 de outubro de 2014 \\ Florianópolis/SC}

CETEC - FUNDAÇÃO CENTRO TECNOLÓGICO DE MINAS GERAIS. Produção de combustíveis líquidos a partir de óleos vegetais: Estudo das oleaginosas nativas de Minas Gerais. Relatório Final do Convênio STI- MIC/CETEC, v. 1, 152 p., 1983.

CICONINI, G.; FAVARO, S. P.; ROSCOE, R.; MIRANDA, C. H. B.; TAPETI, C. F.; MIYAHIRA, M. A. M.; BEARARI, L.; GALVANI, F.; BORSATO, A.V.; COLNAGO, L. A.; NAKA, M. H. Biometry and oil contentes of Acrocomia aculeata fruits from the Cerrados and Pantanal biomes in Mato Grosso do Sul, Brazil. Industrial Crops and Products, v. 45, p. 208214, 2013.

DUARTE, I. D.; ROGÉRIO, J. B.; LICURGO, F. M. da S.; BACK, G. R.; SANTOS, M. C. S.; 82 ANTONIASSI, R.; FARIA-MACHADO, A. F.; BIZZO, H. R.; JUNQUEIRA, N. T. V. Efeito da maturação de frutos de macaúba no rendimento de óleo e na composição em ácidos graxos. In : CONGRESSO DA REDE BRASILEIRA DE TECNOLOGIA DE BIODIESEL, 5.; CONGRESSO BRASILEIRO DE PLANTAS OLEAGINOSAS, ÓLEOS, GORDURAS E BIODIESEL, 8., 2012, Salvador.

INSTITUTO ADOLF LUTZ. Métodos físico-químicos para análise de alimentos. 4. ed. São Paulo: Instituto Adolf Lutz, 2008, 1020 p. Versão eletrônica.

LIRA, F. F.; MACHADO, W.; SANTOS, J. V. F. dos; TAKAHASHI, S. A.; GUIMARÃES, M. de F.; LEAL, A. C. Avaliação da composição centesimal de frutos da macaúba. Biochesmistry and Biotechnology Reports, v. 2, n. 3, p. 17-20, 2013.

MARTINS, A. D. Radiação Gama e secagem na conservação da qualidade do óleo de frutos de macaúba. 2013. 109p. Dissertação (mestrado em Fitotecnia) - Departamento de Fitotecnia, Universidade Federal de Viçosa, Viçosa, 2013.

MOTA, C. S.; CORRÊA, T. R.; GROSSI, J. A. S.; CASTRICINI, A.; RIBEIRO, A. da S. Exploração sustentável da macaúba para a produção de biodiesel: colheita, pós-colheita e qualidade dos frutos. Informe Agropecuário, Belo Horizonte: EPAMIG, v.32, n. 265, p. 41-51, nov./dez. 2011.

OLIVEIRA, A. L. dos S.; TORRES, M. A.; FREIRE, S. J.; PEREIRA, T. B.; SANTOS, T. F. dos; SILVA, V. O. da; AZEVÊDO, L. C. de. Caracterização físico-química da macaúba (Acrocomia Aculeata Jacq. Lodd) cultivada no sertão pernambucano. In: Congresso de Pesquisa e Inovação da Rede Norte e Nordeste Tecnológica, IV, 2009, Belém.

SILVA, G. C. R.; CAÑO ANDRADE, M. H. Development and simulation of a new oil extraction process from fruit of macauba palm tree. Journal of Food Process Engineering. v. x, p. 1-12, 2011.

SOUZA, C. F. T. de. Desenvolvimento, Maturação e Sistemas de Colheita de frutos da macaúba (Acrocomia aculeata). 2013, 90p. Dissertação (mestrado em Biotecnologia) Universidade Católica Dom Bosco, Campo Grande, 2013. 